\title{
Photoconductive Metasurfaces for Terahertz Detection
}

\author{
Lucy Hale, ${ }^{1}$ Thomas Siday, ${ }^{1}$ Polina P. Vabishchevich, ${ }^{2,3}$ C. Thomas Harris, ${ }^{2,3}$ Ting Shan Luk, ${ }^{2,3}$ \\ John F. Reno, ${ }^{2,3}$ Igal Brener, ${ }^{2,3}$ and Oleg Mitrofanov ${ }^{1,2}$ \\ ${ }^{1}$ Electronic and Electrical Engineering, University College London, London, WCIE 7JE UK \\ ${ }^{2}$ Center for Integrated Nanotechnologies, Sandia National Laboratories, Albuquerque, NM 87123 \\ ${ }^{3}$ Sandia National Laboratories, Albuquerque, NM 87185, USA \\ Authore-mail address: o.mitrofanov@ucl.ac.uk
}

\begin{abstract}
We developed terahertz detectors with integrated perfectly-absorbing photoconductive metasurfaces as an active region for improved efficiency and performance. The metasurface switches photoconductivity in the detector with contrast of $\sim 10^{7}$ using substantially reduced $(>10 \mathrm{X})$ optical power.

(C) 2020 The Authors.
\end{abstract}

\section{Introduction}

Photoconductive (PC) switches are among the most common detectors of terahertz (THz) radiation [1], which enable $\mathrm{THz}$ time-domain spectroscopy and imaging. Their performance relies on a combination of optical and electronic properties, which are difficult to achieve using common semiconductor materials [1]. In particular, they require efficient absorption of the optical gating pulse, sub-picosecond photoexcited carrier lifetime and high contrast between its $\mathrm{ON}$ and OFF states. THz photoconductive detectors with plasmonic nanostructures in their active region have been recently introduced in order to achieve more efficient absorption of photoexcitation, primarily near the photoconductor surface [2-4]. However, the plasmonic structures may also undermine the switching contrast and introduce Ohmic losses. Alternatively, perfect absorption of the gating pulse can be achieved using all-dielectric metasurfaces [5,6].

Here, we discuss all-dielectric perfectly absorbing photoconductive metasurfaces based on the concept of two degenerate critical coupled resonators arrayed in a metasurface [7,8]. When integrated into the active region of a photoconductive $\mathrm{THz}$ detector, the metasurface provides the desired optical and electronic properties, and for the first time enables optimal operation of such detectors with sub-mW level of optical excitation [6].

\section{Results}

We designed the perfectly absorbing metasurface following the concept of two degenerate and critically coupled dielectric resonators [7]. For the highest field confinement at the gating wavelength $(\sim 800 \mathrm{~nm})$, we selected the magnetic dipole modes, and we designed and fabricated a network of GaAs cubes (Fig. 1(a,b)), which support magnetic dipole modes $M_{\mathrm{x}}$ and $M_{\mathrm{z}}$. The cubes are electrically connected on one side by bars, which enable conduction of the photoexcited carriers between the THz detector electrodes (Fig. 1(c)). At the same time the bars break the metasurface symmetry and enable direct excitation of the magnetic dipole mode $M_{\mathrm{z}}$, which is dark in symmetric resonators [9]. Simultaneous excitation of the two modes leads to perfect absorption of light around 800nm (Fig. 1(d)).
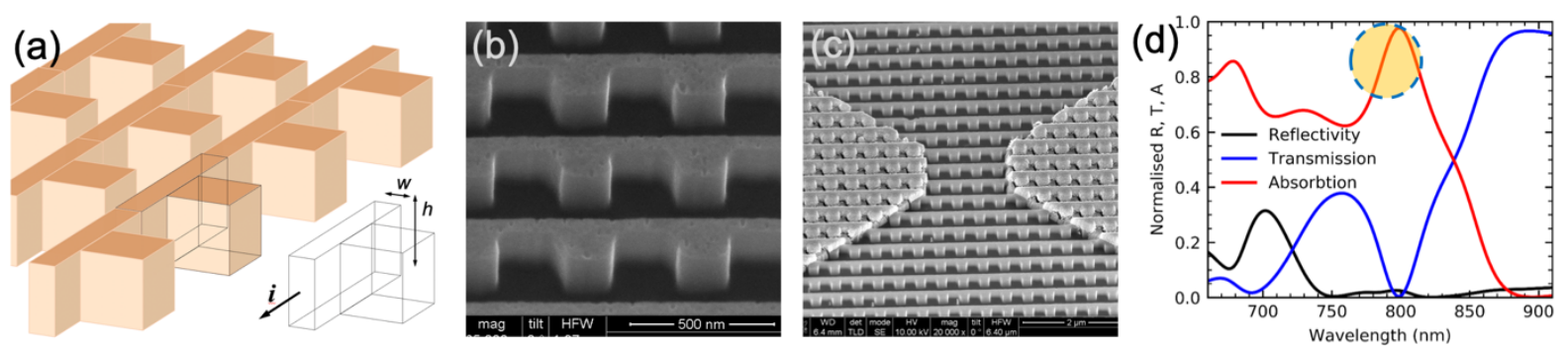

Fig. 1. (a) Schematic diagram of the metasurface. (b) SEM image of the GaAs metasurface showing resonator cubes and the connecting bar. (c) The bars extend between two electrodes of the PC THz detector enabling the photocurrent flow. (d) Simulated Absorption, Reflectivity and Transmission of the structure shown in (a). Perfect absorption is achieved at $\sim 800 \mathrm{~nm}$. 

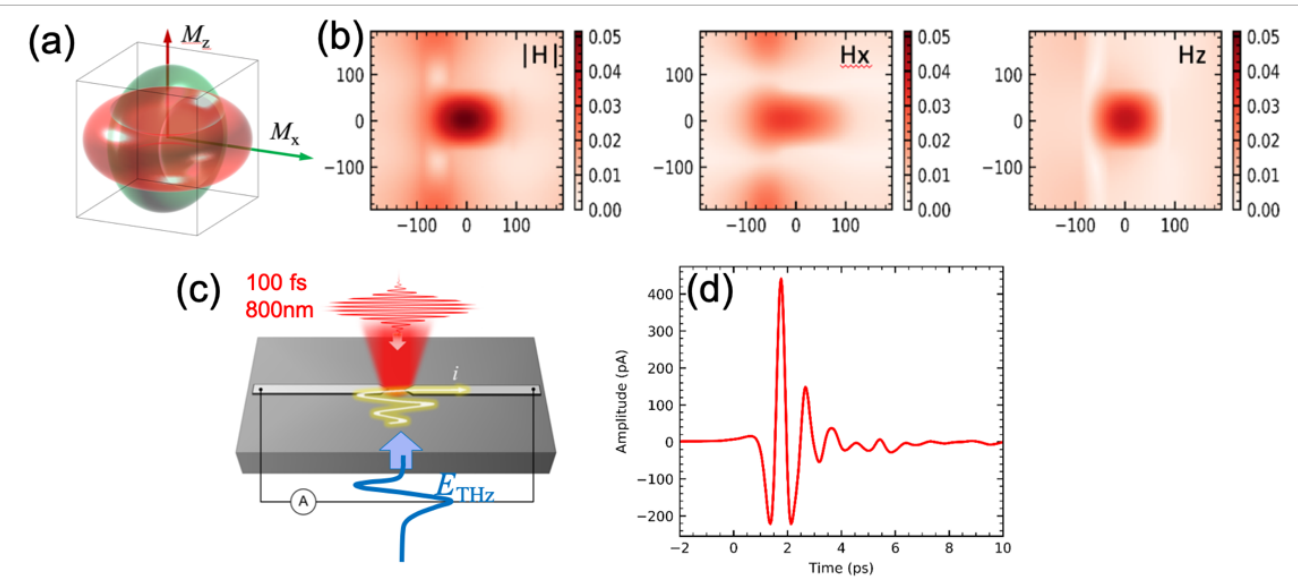

Fig. 2. Schematic illustration of the magnetic dipole modes in a cubing resonator. (b) Simulated magnetic field distribution in the unit cell of the perfectly absorbing structure at $800 \mathrm{~nm}$ : absolute value $|H|$, and two main components $H_{\mathrm{x}}$ and $H_{\mathrm{z}}$. (c) Schematic illustration of a photoconductive $\mathrm{THz}$ antenna detector; and (d) $\mathrm{THz}$ pulse waveform measured using a photoconductive detector with the integrated metasurface.

Figure 2(a,b) shows that following optical excitation at $800 \mathrm{~nm}$ at normal incidence, dipolar distributions of the magnetic field are formed in the resonator for two components, in the $x$ and $z$-directions, indicating that two magnetic dipole modes, $M_{\mathrm{x}}$ and $M_{\mathrm{z}}$, are excited. More detailed analysis of the mode composition in the metasurface can be found in [6]. Resonant frequencies for both modes can be tuned by scaling the structure.

We integrated the developed metasurface into the photoconductive gap of a $\mathrm{THz}$ antenna detector as illustrated in Figs. 1(c) and 2(c). The metasurface enables perfect absorption of the gating optical pulses despite being optically thin $(200 \mathrm{~nm})$. It is also achieved without the use of plasmonic structures. The detector allows us to detect THz pulses (Fig. 2(d)) and reach a maximum signal to noise ratio of $>10^{6}$ (in power) with the detection bandwidth of $\sim 3 \mathrm{THz}$ using an unprecedently low level of optical power, $100 \mu \mathrm{W}$.

\section{Summary}

We developed photoconductive all-dielectric metasurfaces for $\mathrm{THz}$ detectors. The metasurface enables perfect absorption of the gating optical pulse and serves as the active element of the photoconductive switch. We achieved very high switching contrast while using relatively low optical powers of $\sim 100 \mu \mathrm{W}$. THz detectors with the integrated metasurface display a signal to noise ratio of $>10^{6}$ and a detection bandwidth of $\sim 3 \mathrm{THz}$, opening doors to wider use of photoconductive $\mathrm{THz}$ detectors.

\section{Acknowledgements}

This work was supported by the U.S. Department of Energy, Office of Basic Energy Sciences, Division of Materials Sciences and Engineering. Device fabrication was performed at the Center for Integrated Nanotechnologies, an Office of Science User Facility operated for the U.S. Department of Energy (DOE) Office of Science. Sandia National Laboratories is a multi-mission laboratory managed and operated by National Technology and Engineering Solutions of Sandia, LLC., a wholly owned subsidiary of Honeywell International, Inc., for the U.S. Department of Energy's National Nuclear Security Administration under contract DE-NA-0003525. This article describes objective technical results and analysis. The views expressed in the article do not necessarily represent the views of the U.S. DOE or the United States Government.

\section{References}

[1] E. Castro-Camus, M. Alfaro, "Photoconductive Devices for Terahertz Pulsed Spectroscopy: A Review." Photonics Res. 4, A36 (2016).

[2] N.T. Yardimci, M. Jarrahi, "Nanostructure-Enhanced Photoconductive Terahertz Emission and Detection." Small 14 (44), 1802437 (2018).

[3] S. Lepeshov et al., "Boosting Terahertz Photoconductive Antenna Performance with Optimised Plasmonic Nanostructures." Sci. Rep. 8(1), $6624(2018)$.

[4] O. Mitrofanov et al., ASC Photonics 2, 1763 (2015).

[5] O. Mitrofanov et al., "Efficient Photoconductive Terahertz Detector with All-Dielectric Optical Metasurface." APL Photonics 3, 51703

(2018).

[6] T. Siday et al., "Terahertz Detection with Perfectly-Absorbing Metasurface.” Nano Lett. 19, 2888-2896 (2019).

[7] J.R. Piper, V. Liu, S. Fan, “Total Absorption by Degenerate Critical Coupling." Appl. Phys. Lett. 104(25), 251110 (2014).

[8] X. Ming et al., "Degenerate Critical Coupling in All-Dielectric Metasurface Absorbers." Opt. Express 25(20), 24658 (2017).

[9] S. Campione et al., "Broken Symmetry Dielectric Resonators for High Quality Factor Fano Metasurfaces." ACS Photonics 3(12), 2362

(2016). 